\title{
Tears of detached retinal pigment epithelium
}

\author{
A. HOSKIN, A. C. BIRD, AND K. SEHMI
}

From the Institute of Ophthalmology Professorial Unit, Moorfields Eye Hospital, City Road, London ECV1 2PD

SUMMARY Ripping of detached pigment epithelium appears to be a common sight-threatening complication of pigment epithelial detachments and occurs at the junction of the detachment and flat pigment epithelium. The characteristics of the detachment prior to the rip suggest that the tear occurs in pigment epithelium which is detached without its basement membrane. The tear is followed by retraction of the pigment epithelium, revealing bare Bruch's membrane. The defect may remain apparently unaltered, or may be recovered by normal looking pigment epithelium, but most commonly is replaced by a fibrous plaque. Most patients had a profound reduction in visual acuity.

Detachment of the pigment epithelium is a well recognised clinical entity within the spectrum of age-related macular degeneration. ${ }^{1}$ So far it has been considered that the major complication of such a lesion is invasion of the subpigment epithelial space by new blood vessels derived from the choroid, resulting in a vascular disciform lesion. ${ }^{1-4}$

During the past 2 years we have recognised an additional complication of pigment epithelial detachments which also results in loss of visual acuity. This consists of a rip in the detached pigment epithelium and subsequent retraction of that layer.

\section{Patients and Methods}

During a 2-year period tearing of detached pigment epithelium has been identified in 44 eyes of 36 patients. All patients were seen in the retinal diagnostic department of Moorfields Eye Hospital and were studied by fluorescein fundus angiography. The average age was 69 years (range 54-85).

\section{Results}

P RE - T E A R C H A R A C TER I S T I C S

Twelve eyes were examined in which a pigment epithelial detachment was seen before and after ripping of the pigment epithelium. In 8 the original appearance of the detachment differed from one part to another (Figs. 1, 2). In one segment of the lesion biomicroscopy showed that the detached pigment epithelium had drusen on its deep surface and, on angiography, the hyperfluorescence was uneven owing to irregular transmission of light at the level of the pigment epithelium. In the other area the detached pigment epithelium was relatively featureless clinically; angiographically the lesion was hypofluorescent early, and later the hyperfluorescence was uniform. The latter portion was often more

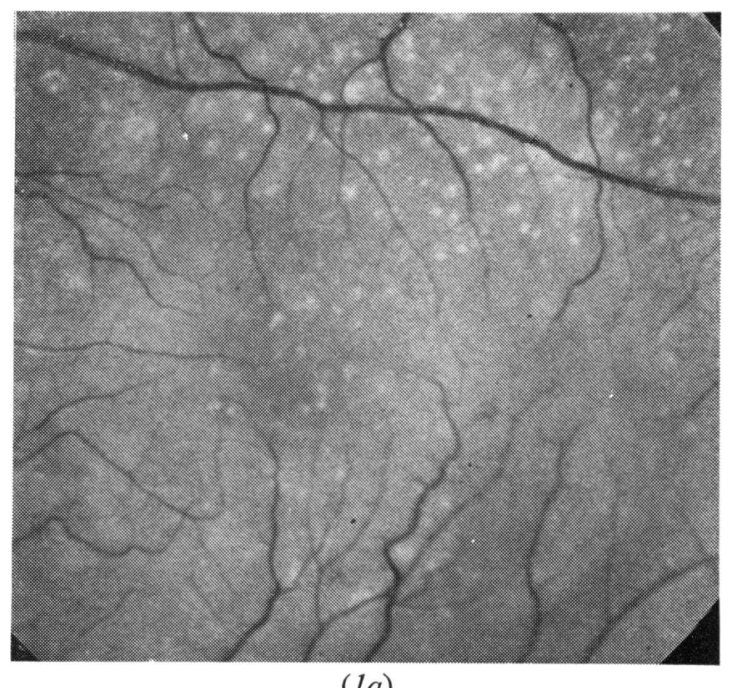

(1a)

Fig. 1 (1a) Left eye of a 70-year-old male, showing detachment of the pigment epithelium with drusen in the central portion only. Fluorescein angiography shows irregularity of transmission in the central area. 


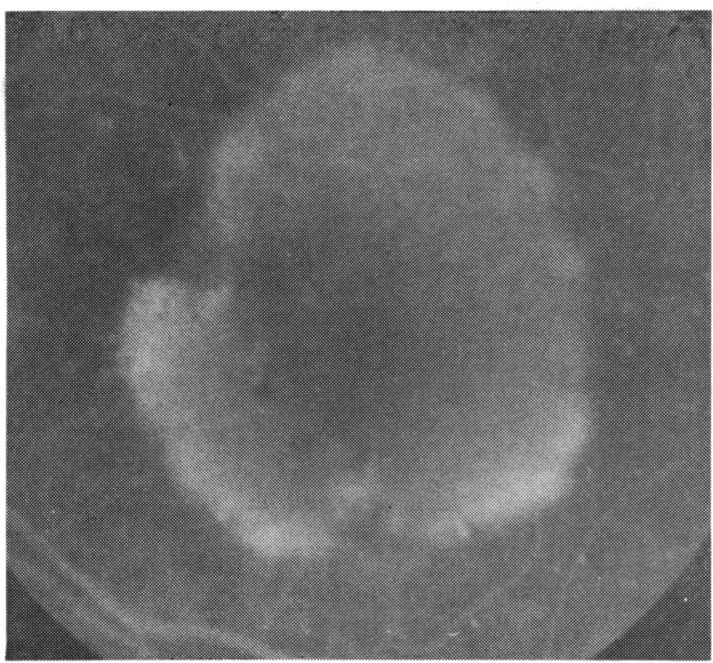

$(1 b)$

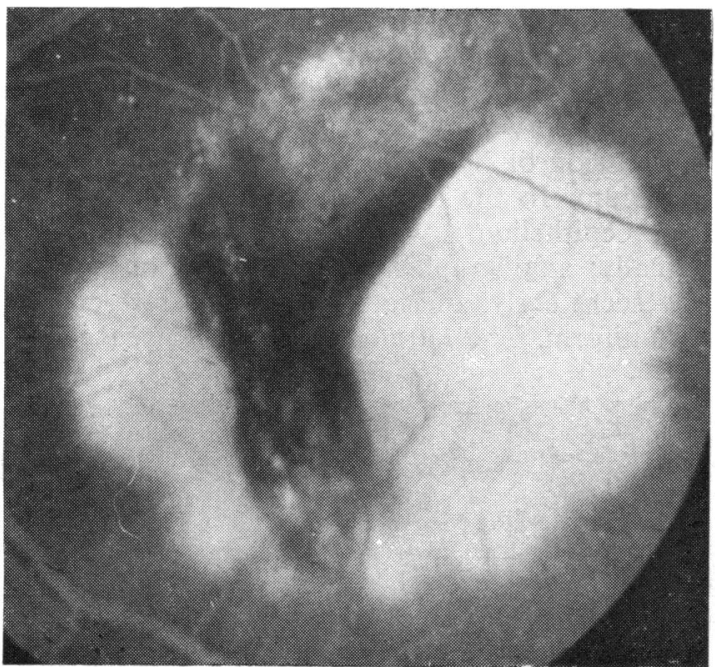

(1d)

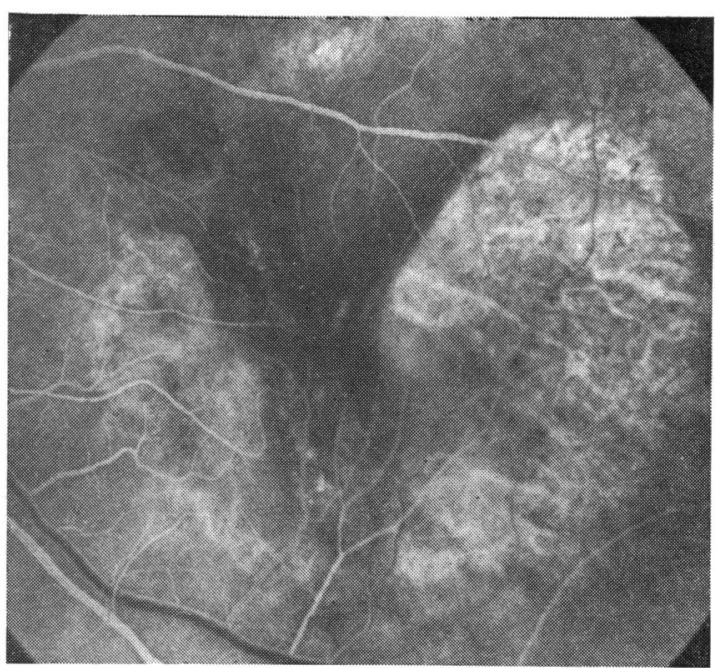

(1c)

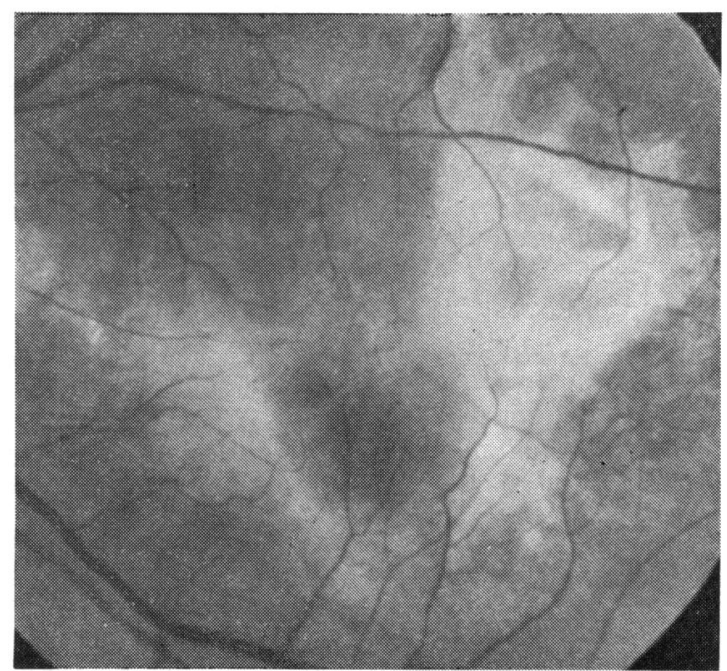

(1e)

(1b) (1c,d) (1e) 3 months later the patient suffered visual loss (6/60), and angiography showed tearing of the pigment epithelium at the lateral and medial margin of the detachment. Within 6 months the bare Bruch's membrane was covered by fibrous material.

highly detached from Bruch's membrane than the former. In such lesions the rip occurred along the edge of the featureless and evenly hyperfluorescent portion of the lesion. In 4 of the remaining patients the whole of the lesion was evenly hyperfluorescent (Fig. 3).

\section{P A T TER N OF TEA R}

The tear virtually always occurred at the edge of the pigment epithelial detachment and the remaining detached pigment epithelium re- tracted, leaving an area of bare Bruch's membrane (Figs. 1-4). The overlying retina remained intact. The retracted pigment epithelium was often thrown into fine folds parallel to the edge of the rip, and this edge remained free in the subpigment epithelial space for a few days (Fig. 4). Thereafter in most cases it became reattached to Bruch's membrane at a new site, giving rise to a smaller and darker pigment epithelial detachment, with a well defined area of bare Bruch's membrane at its side. In a few 


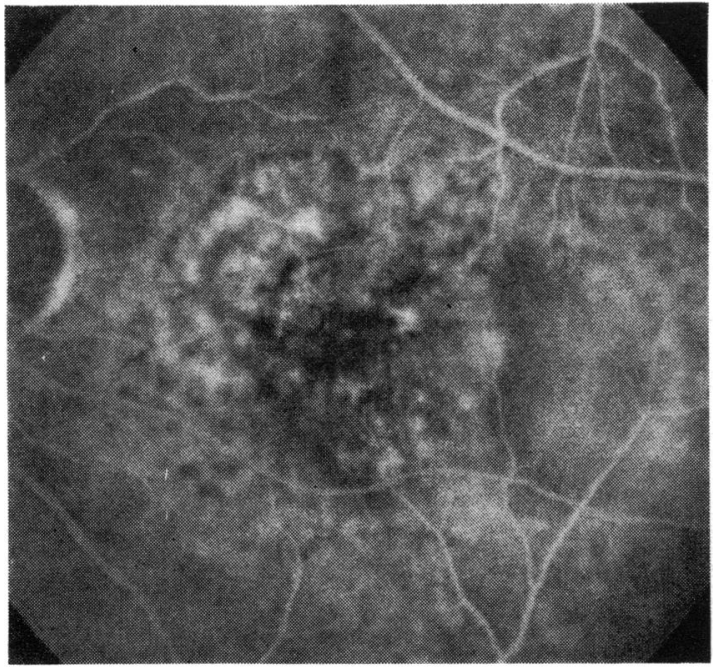

(2a)

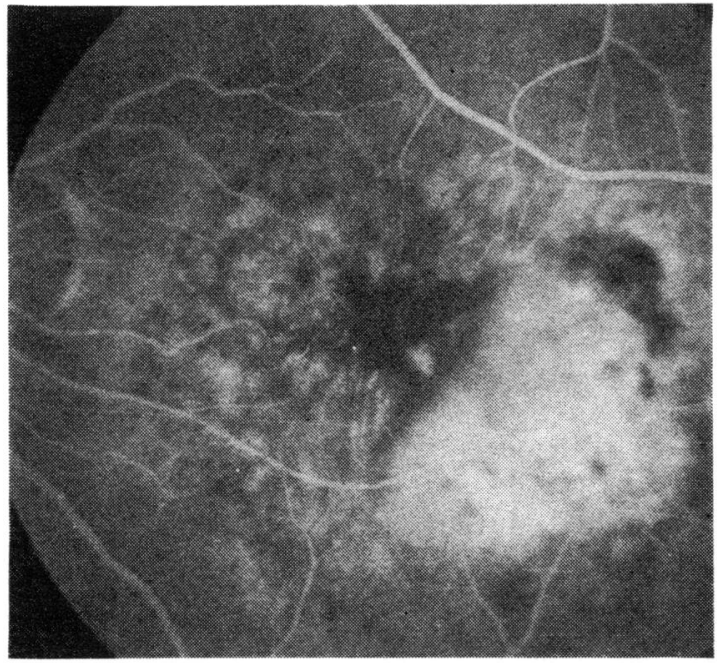

(2c)

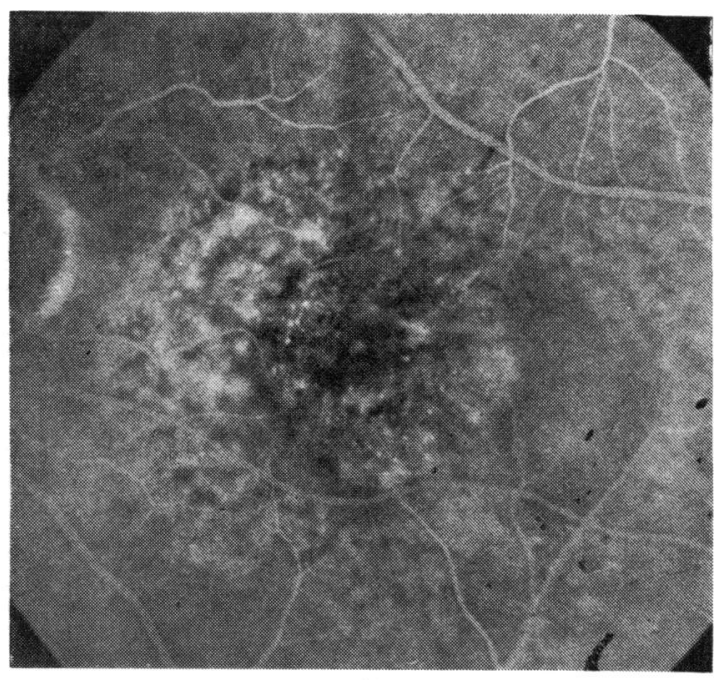

(2b)

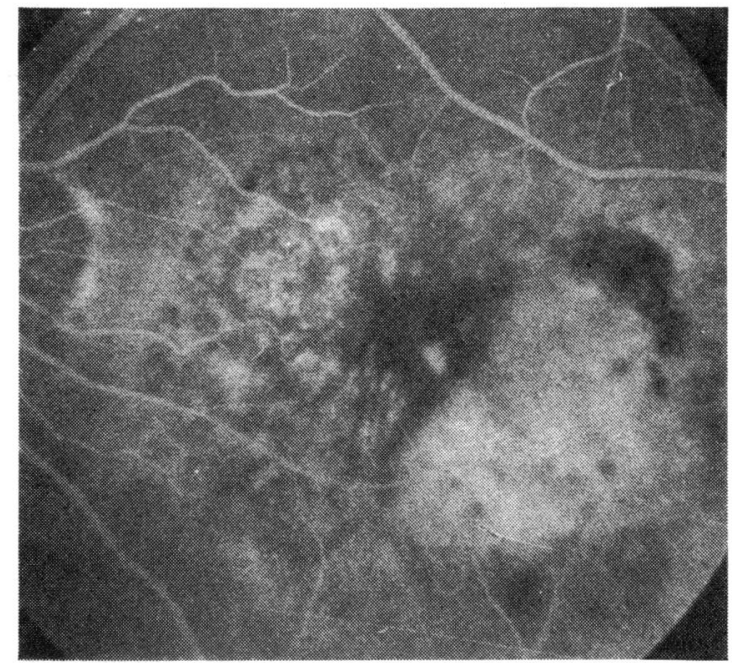

(2d)

Fig. 2 (2a,b) Fluorescein angiogram of the left eye of a 72-year-old female, showing detachment of the pigment epithelium in 2 parts. The medial half has irregular transmission of fluorescein, while the temporal half shows even fluorescence. (2c,d) Within 4 months visual acuity fell to 6/36. Fluorescein angiography showed tearing of the pigment epithelium along the temporal edge of the detachment. A stereo view may be obtained by converging while viewed at a distance of $2 \mathrm{ft}(60 \mathrm{~cm})$.

patients the pigment epithelium flattened spontaneously and in one the pigment epithelium edge curled up so that it remained free in the subretinal space. In 5 patients the rip was accompanied by bleeding into the subretinal space, although no subretinal blood vessels were detected by fluorescein angiography as the blood reabsorbed.

At this stage the appearance of the fluorescein angiogram was characteristic (Figs. 1-4). In the area of bare Bruch's membrane the choroidal vessels were seen in the first frame, being replaced by even hyperfluorescence within 2 seconds. The dye appeared to be at the level of Bruch's membrane and did not appear to leak into the subretinal fluid in most cases. In several there was some late dye accumulation subretinally. The free edge of the pigment epithelium 


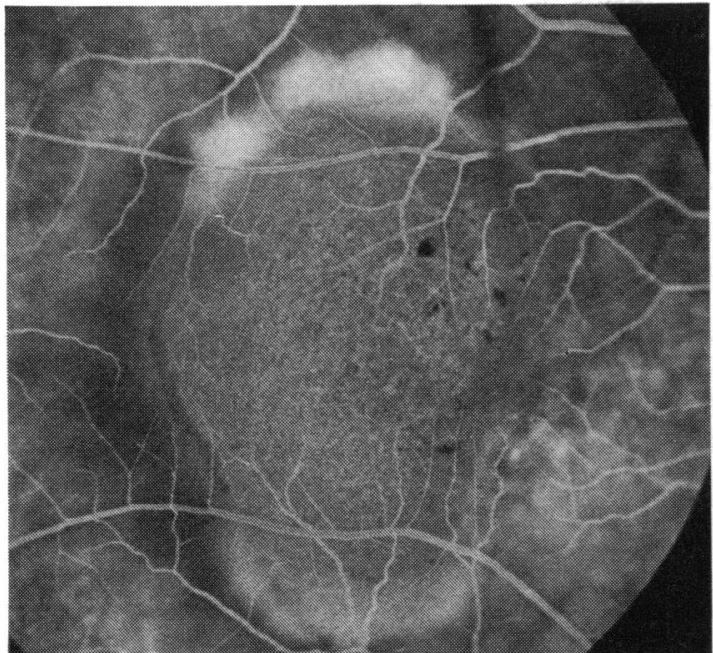

(3a)

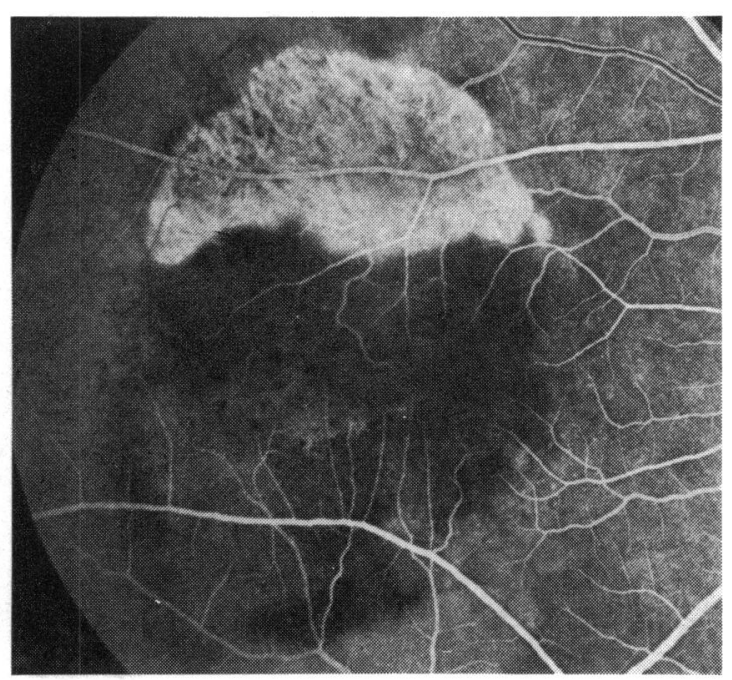

$(3 b)$

Fig. 3 (3a) Fluorescein angiogram of the right eye of a 56-year-old female, showing a relatively featureless pigment epithelial detachment with some leakage from the superior edge into the subretinal space. (3b) Photocoagulation was undertaken and 2 weeks later the pigment epithelium had torn along the upper boarder of the lesion.

was well demarcated, and the residual detached pigment epithelium was dark and folds were seen in some (Fig. 4).

Many of the patients reported a sudden change in vision at the time of the rip if the subfoveal pigment epithelium was involved. In one case observed closely during the week after the event increase in distortion was the initial symptom, but a visual acuity of $6 / 9$ was main-

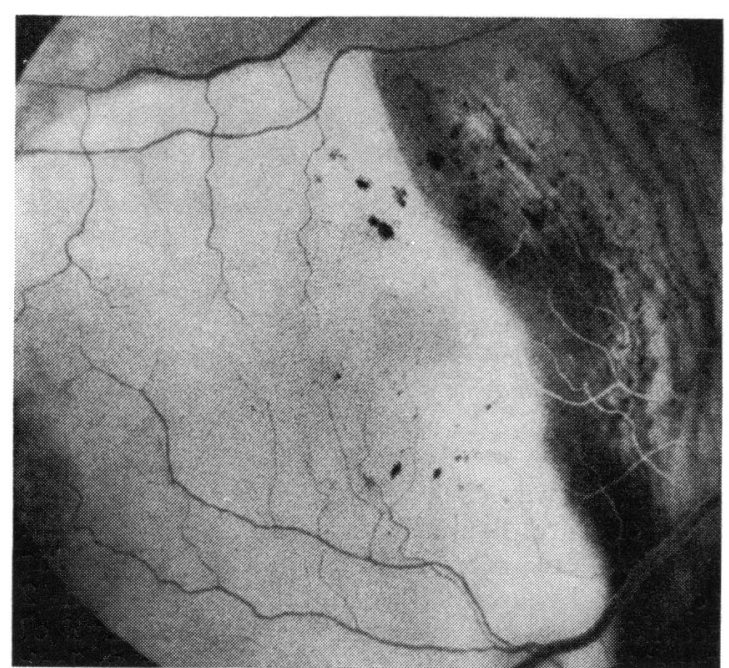

Fig. 4 Fluorescein angiography of the right eye of a 68-year-old female immediately after tearing of the pigment epithelium, showing folding of the detached pigment epithelium. tained for 4 days despite baring of the foveolar receptors by pigment epithelium. On the fifth day visual acuity was $6 / 60$. Of the 44 eyes 26 had a visual acuity of $6 / 60$ or worse.

\section{P O S T - T E A R R E P A I R}

In 4 patients the area of bare Bruch's membrane remained unchanged during the period of review. In 2 the area became covered by normal looking pigment epithelium derived from the edge of the defect not involved in the original detachment. In the remaining eyes the defect became covered by fibrous tissue which at times acquired a vascular content, but it was rarely prominent and vascularisation did not precede the fibrosis (Fig. 1d).

\section{Discussion}

Our experience suggests that tearing of detached pigment epithelium is a common phenomenon and carries a high risk to visual acuity.

In patients seen prior to ripping of the pigment epithelium the area in which the rip occurred had distinct morphological characteristics. Some pigment epithelial detachments had an even, late hyperfluorescence, which contrasted with the other segment in which hyperfluorescence was early and irregular. These observations call into question the site of separation of the detached pigment epithelium from Bruch's membrane. Two potential planes of cleavage exist, namely, 
between the basement membrane of the pigment epithelium and the remainder of Bruch's membrane, and between the pigment epithelial outer cell membrane and its own basement membrane. Both these planes are subject to accumulation of abnormal material as an age-related process. Drusen result from deposition of debris within Bruch's membrane and tend to displace the pigment epithelial basement membrane from the inner collagenous layer of Bruch's membrane. The basal linear deposit described by Sarks displaces the pigment cell from its original basement membrane. ${ }^{5}$ o

The observation of uneven hyperfluorescence of the pigment epithelial detachment suggests the presence of a layer on the outer surface of the pigment epithelium, giving rise to irregular transmission of light. This in turn suggests that at least in some pigment epithelial detachments the plane of separation is such that the pigment epithelium becomes detached together with its basement membrane, which itself may be thickened or reduplicated with drusen impacted in that membrane. By contrast, the even hyperfluorescence may indicate a lesion in which the cleavage plane is between the pigment epithelium and its basement membrane. Our observations suggest that both these circumstances exist and may occur within the same pigment epithelial detachment. Predictably, presence or absence of basement membrane and other material on the inner surface of the pigment epithelium may modify the mechanical properties of the detached tissues. In pigment epithelial detachments in which both circumstances existed in the same lesion, the segment that detached without its basement membrane was usually more highly elevated. Our observations imply that, when the pigment epithelium ripped, it did so in an area in which we presume the pigment epithelium to be detached from its basement membrane.

It is significant that tears in the pigment epithelium have not been observed in young patients. This might be predicted, since the basal linear deposit is restricted to the elderly, and therefore the pigment epithelium in the young could be expected to detach with its basement membrane.

The early stages of pigment epithelial rip were seen in a number of patients, and the tear occurred initially at the edge of the pigment epithelial detachment. In a minority of cases ripping of the pigment epithelium was accompanied by haemorrhage into the subpigment epithelial and subretinal spaces. This observation suggests that these lesions might contain sub- pigment epithelial neovascular tissue and that the haemorrhage was derived from these vessels. However, in the majority of lesions in which haemorrhage occurred at the time of tearing, subpigment epithelial neovascular tissue could not be identified once the haemorrhage resolved. This does not preclude the possibility of bleeding from a flat neovascular complex on the inner surface of Bruch's membrane, which closes after the tear. An alternative source of the haemorrhage could be the choriocapillaris. Normal Bruch's membrane is highly resistant to rupture, but release of tangential stress may occur at the time of pigment epithelial tearing, which in turn may be sufficient to disrupt abnormal Bruch's membrane with the consequent disturbance of choroidal blood vessels. Within days of the rip the free edge of the pigment epithelium became reattached to Bruch's membrane, forming a smaller epithelial detachment in most patients, and subsequently became flat in some.

The nature of the repair process varied. In the majority of cases the defect in the retinal pigment epithelium was replaced by a plaque of fibrous tissue, which could become vascular, but the blood vessel content of the tissue was rarely prominent in the early stages of repair. The process is quite different from that seen in primary vascular disciform lesions in which invasion of the subretinal space by blood vessels from the choroid represents the primary event and fibrosis is only a secondary process which accompanies closure of the vessels. In a few lesions the inner surface of Bruch's membrane became re-covered by a layer of relatively normal looking pigment epithelium. In the remaining patients the appearance did not alter after the initial rip, suggesting that the Bruch's membrane remained denuded.

The pattern of the healing process appears to be analogous to that seen after experimental trauma to the pigment epithelium. In small lesions the defect is covered by cells sliding over the area with limited division of pigment epithelial cells. $^{7}$ In larger defects the pigment epithelial cells proliferate and become fibroblastic. ${ }^{8}$

Ripping of detached pigment epithelium had severe consequences to the visual acuity of most patients. The moment of ripping was often accurately timed by the patient. This is probably due to the interference by blood of metabolic exchange between retinal receptors and pigment epithelium of the fovea, or by retraction of the pigment epithelium away from the fovea. In the patient seen 4 days after the rip the visual 
acuity of $6 / 9$ fell to $6 / 60$ within a further 3 days.

This time sequence of receptor dysfunction following interruption of the metabolic relationship between it and the pigment epithelial cells conforms with structural changes identified experimentally ${ }^{9}$ (and J. Marshall, personal communication). In only 8 out of 44 eyes did ripping of the pigment epithelium leave the relationship between the foveal receptors and the pigment epithelial cells intact, and in those patients relatively good central visual acuity was maintained.

The recognition of ripping of detached pigment epithelium has led to observations which allow reinterpretation of certain fundus lesions. In some cases the appearance of the fresh rip has lead to the mistaken impression that there was invasion of the subpigment epithelial space by choroidal blood vessels, which in turn induced the clinician to undertaken photocoagulation. The presence of subretinal fibrous tissue in a lesion is often interpreted as good evidence that the primary lesion was a vascular disciform lesion. However, fibrosis which may occur after a rip implies an alternative pathogenesis. Finally, Bruch's membrane exposed by tearing of the pigment epithelium has been interpreted as geographic atrophy. ${ }^{1011}$

The ripping of pigment epithelium following laser photocoagulation is also relevant to therapy. In 7 patients who had tearing of the pigment epithelium immediately after laser photocoagulaticn the site of the rip could have been predicted before photocoagulation was applied, and this complication of treatment of the pigment epithelial detachment might have been avoided.

A. H. was supported by the A. E. Baker grant (Canadian National Institute for the Blind).

This study was supported in part by the Medical Research Council (G976/618).

\section{References}

1 Gass JDM. Pathogenesis of disciform detachment of the neuroendothelium III. AmJ Ophthalmol 1967; 63:573-711.

2 Meredith TA, Braley RE, Aaberg TM. Natural history of serous detachments of the retinal pigment epithelium. Am J Ophthalmol 1979; 88:652-60.

3 Braunstein RA, Gass JDM. Serous detachments of the retinal pigment epithelium in patients with senile macular disease. Am J Ophthalmol 1979; 88:652-60.

4 Klein ML, Obertynski H, Patz A, Fine SL, Kimi M. Follow-up of detachment of the retinal pigment epithelium. Br J Ophthalmol 1980; 64:412-6.

5 Sarks SH. Ageing and degeneration in the macular region-a clinical-pathological study. $\mathrm{Br} J$ Ophthalmol $1976 ; 69: 324-41$.

6 Sarks SH. Drusen and their relationship to senile macular degeneration. Aus J Ophthalmol 1980; 8:117-30.

7 Marshall J, Mellerio J. Disappearance of the retroepithelial scar tissue from ruby laser photocoagulation. Exp Eye Res $1971 ; 12: 173-4$.

8 Marshall J, Mellerio J. Laser irradiation of retinal tissue. Br Med Bull 1970; 26:156-60.

$9 \mathrm{Kroll} \mathrm{AJ,} \mathrm{Machemer} \mathrm{R.} \mathrm{Experimental} \mathrm{detachment} \mathrm{in} \mathrm{the}$ owl monkey III. Electron microscopy of retina and pigment epithelium. Am J Ophthalmol 1968; 66:410-27.

10 Gass JDM. Stereoscopic Atlas of Macular Diseases. St Louis: Mosby, 1970.

11 Blair CJ. Geographic atrophy of the retinal pigment epithelium. Arch Ophthalmol 1975; 93:19-25. 